Mots. Les langages du politique

\title{
Politique, humour et campagne électorale. Les enjeux d'une politique-spectacle
}

\author{
Maria-Aldina Marques
}

\section{(2) OpenEdition \\ Journals}

Édition électronique

URL : https://journals.openedition.org/mots/21146

DOI : $10.4000 /$ mots. 21146

ISSN : 1960-6001

Éditeur

ENS Éditions

Édition imprimée

Date de publication : 22 avril 2013

Pagination : 61-75

ISBN : 978-2-84788-393-0

ISSN : 0243-6450

Référence électronique

Maria-Aldina Marques, « Politique, humour et campagne électorale. Les enjeux d'une politiquespectacle ", Mots. Les langages du politique [En ligne], 101 | 2013, mis en ligne le 22 avril 2015, consulté le 22 avril 2022. URL : http://journals.openedition.org/mots/21146 ; DOl : https://doi.org/10.4000/ mots. 21146

\section{(c) ENS Éditions}




\section{Politique, humour et campagne électorale. Les enjeux d'une politique-spectacle ${ }^{\star}$}

L'influence des médias dans le monde de la politique n'est évidemment pas une nouveauté. En fait, l'histoire de l'influence grandissante de la télévision sur la vie politique portugaise est déjà longue. L'ancien président de la République portugaise, Mário Soares, le soulignait déjà en 1997, dans un programme de télévision sérieux, en établissant un lien essentiel entre démocratie et médias : "Os políticos que não passam bem na televisão dificilmente se poderão impor nas democracias modernas. » (Mário Soares, O Século do Povo, 26 novembre 1997) ${ }^{1}$

Néanmoins, un événement singulier dans le panorama politique portugais a marqué la campagne pour les élections législatives de 2009 : plusieurs leaders politiques ont participé à une émission humoristique sur la chaîne de télévision SIC en tant qu'invités d'un groupe de quatre comédiens appelé Gato Fedorento [Chat Puant]. Tous les leaders politiques des partis qui siègent au parlement, qu'ils soient de droite ou de gauche, y ont participé, de même que les candidats aux législatives, les maires, les anciens leaders politiques, entre autres.

Dès lors, la singularité de cet événement se trouve dans l'adhésion qu'il a suscitée de la part des leaders des différents partis politiques, ainsi que dans son succès auprès des citoyens; mais ses conséquences sont particulièrement importantes au niveau des rapports des politiques et de la politique aux programmes télévisuels de divertissement (McNair, 2011).

La spécificité politique d'une campagne électorale reliée à ce pouvoir croissant de la télévision dans la construction de l'agenda public, contribue certainement à l'apparition et à la portée de ce type de phénomènes, mais ne les délimite pas. Dans cette perspective, cette émission de télévision est typique d'une situation où se jouent des influences mutuelles, en fonction de la conjonction d’intérêts politiques et médiatiques socialement déterminants.

Je remercie Michèle Monte pour sa relecture attentive et pertinente de mon article.

1. «Les politiques qui ne passent pas bien à la télévision pourront difficilement s'imposer dans les démocraties modernes. » (Ma traduction, de même que pour tous les autres extraits traduits du portugais dans cet article.)

Universidade do Minho, Portugal

mamarques@ilch.uminho.pt

Mots. Les langages du politique $\mathrm{n}^{\circ} 101$ mars $2013 \bullet 61$ 
Une campagne électorale est un moment décisif des systèmes démocratiques, les élections étant la voie unique d'accès au pouvoir. Si seul le peuple a le pouvoir d'élire, il faut que les politiques reviennent au peuple, le prennent comme destinataire premier et principal de leurs discours de campagne. Les différentes activités programmées et réalisées pendant cette période ont comme objectif fondamental de renforcer l'appui politique, de séduire les citoyens, de les faire voter pour soi. D'ailleurs, elles prennent le devant de l'actualité nationale; tous les médias les amplifient, les diffusent, les font circuler. La campagne électorale est un phénomène à la fois politique et social.

Discursivement, c'est un macro-événement hétérogène, surdéterminé par cet appel au vote et par un rapport direct et étroit avec les citoyens. Le discours électoral est un discours de propagande ${ }^{2}$, constitué de pratiques discursives variées. La proximité avec le peuple, ou mieux, avec les masses populaires, l'ambiance d'euphorie et de mission politique et sociale, imposant aux leaders politiques de parcourir le pays d'un bout à l'autre dans des actions de rue, des meetings et des rassemblements divers pour appeler au vote, rendent ce discours potentiellement populiste (Charaudeau, 2011). Les partis politiques sont en pleine campagne et les résultats se jouent notamment et de plus en plus sur l'image des candidats. Or, les moyens de communication sociale sont essentiels à cette visibilité personnelle. N’importe quelle émission médiatique semble être bonne pour faire circuler le message, et le politique s’impose aux médias.

À leur tour, les moyens de communication sociale, et la télévision en particulier, accompagnent chaque moment de cette mise en scène politique, à laquelle les journaux télévisuels accordent une place centrale. Et leur pouvoir est justement là, dans le choix de ce qu'il convient de montrer à la télévision, ou des événements qu'il faut créer : depuis les sondages, les interviews, jusqu'aux débats, si importants en termes de campagne électorale et si rentables économiquement. Le cercle d'influences se noue et l'agenda politique est directement conditionné par l'agenda des médias³. Autrement dit, les médias s'imposent à la politique.

\section{Politique et humour : amuser pour mieux communiquer}

La politique est une affaire dite sérieuse, en tant qu'elle s'occupe de la vie publique du pays. Cependant l'humour, bien qu'épisodique et contrôlé, n'est pas absent des discours politiques. C'est le cas, par exemple, des débats parlementaires, où l'humour sert une stratégie de confrontation et de construction

2. Sur les caractéristiques définissant ce genre de discours, nous renvoyons à Charaudeau, 2010.

3. McNair (2011, p. 43), sous le titre Political Media, écrit : «The media, or those who work in them, should be viewed as important political actors in themselves. » 
d'un ethos d'homme d'esprit intellectuellement stimulant4. D'un autre côté, et traditionnellement, l'humour vit des politiques. Dans ce dernier cas, le rapport de la politique à l'humour est asymétrique, les politiques sont la cible de l'humour au profit d'un public qui veut s'amuser. Or, l'amplification sociale et surtout médiatique de la sphère politique et de l'image des politiques pendant la campagne électorale attire forcément l'attention des comédiens dont les politiques sont la cible la plus prévisible, surtout dans le cadre du divertissement télévisuel. Malgré cela, les politiques semblent être convaincus de l'importance de la télévision, qui reste un moyen de communication attirant pour tous ceux qui sont engagés dans ce macro-acte de séduction électorale. En fait, ils envisagent la relation à cette télévision d'une façon positive ou du moins inévitable, comme s'il fallait absolument amuser pour mieux communiquer et séduire. Le Premier ministre en fonction en 2011 le soulignait en caractérisant sa participation à un programme télévisuel de divertissement comme un impératif politique : "Estou em campanha e tenho essa obrigação de vir a estes programas. » (José Sócrates, Só Visto, 15 mai 2011)5

À ce niveau, ce n'est plus un simple rapport de la politique à l'humour qu'il faut considérer, c'est le rapport d'une certaine façon de faire de la politique aux médias télévisuels, ayant des implications sociales plus profondes, qu'il faut questionner davantage.

\section{Données. Cadre théorique. Objectifs}

O Gato Fedorento esmiúça os sufrágios [Le Chat Puant épluche le scrutin] est une émission de télévision humoristique, une sorte de talk-show réalisé pendant la campagne électorale pour les élections législatives de 2009 au Portugal6.

L'émission dure une demi-heure environ. La première partie du programme est la critique comique, moqueuse, des faits de la journée de campagne; la deuxième, qui constitue le corpus de notre analyse, est une interaction verbale entre un comédien et un politique. Le temps imparti à chacune d'elles est de dix minutes environ. Ces rencontres ont eu lieu du 14 septembre au 23 octobre 2009. Les données sélectionnées sont constituées de cinq interactions auxquelles participent les leaders politiques des partis qui siègent au Parlement

4. Tsakona (2009, p. 230) affirme : «Humour appears to be an important aspect ofleadership. » Sur l'humour au parlement portugais voir aussi Marques (2005).

5. "Je suis en campagne électorale et je suis donc obligé de participer à ce genre de programmes de télévision.»

6. La presse n'a fourni que peu de renseignements sur ce programme humoristique annoncé un peu partout. Il est difficile, sinon impossible, d'identifier la situation globale où il s'est construit, et notamment de rendre compte des négociations entre tous les intéressés. Selon Ricardo Pereira, l'un des comédiens, le script du programme $O$ Gato Fedorento esmiúça os sufrágios suit le modèle de talk-show intitulé The Daily Show, que présente Jon Stewart sur la chaine américaine CBS. Mais en ce qui concerne l'humour, l'influence des Monty Python est évidente. 
(de droite à gauche, respectivement : Paulo Portas, CDS-PP (Partido do centro democrático e social - Partido popular); Manuela Ferreira Leite, PSD (Partido social democrata); José Sócrates, PS (Partido socialista); Jerónimo de Sousa, PCP (Partido comunista português) et Francisco Louçã, BE (Bloco de Esquerda)7. En 2009, le Portugal est déjà en crise économique et sociale. Le Parti socialiste est au pouvoir, mais il va perdre la majorité dans ces élections. Le combat politique s'est révélé vif et agressif.

Nous portons notre attention sur les spécificités discursives qu'impose ce rapport risqué de la politique à l'humour. Dans le cadre de l'analyse linguistique des discours, et des discours en interaction spécifiquement ${ }^{8}$, nous nous proposons d'analyser la façon dont la construction interactionnelle est gérée et négociée par les interlocuteurs, étant donné ces contraintes politiques et médiatiques globales. Notre hypothèse est que les fonctionnements discursifs et langagiers mis en scène par les interlocuteurs, selon les objectifs qu'ils poursuivent et les places qu'ils occupent dans l'interaction, éclairent la façon dont les politiques négocient les rapports aux médias et au peuple portugais et cogèrent leurs interventions pour atténuer les risques et optimiser les bénéfices. À la suite de Vion (1992, p.112), notre perspective est que les relations interpersonnelles sont le point d'ancrage de ce procès interactionnel, car « on ne peut espérer communiquer sans établir un positionnement réciproque ».

\section{Des raisons politiques à une rencontre humoristique}

Cette émission humoristique procède d'une convergence d'intérêts, mais les motivations sous-jacentes à ce rassemblement sont fort diverses. Pour les comédiens, il s'agit de réussir un programme d'humour en direct qui amplifie et ridiculise des situations de campagne, c'est-à-dire qu'ils se livrent à une critique moqueuse, caustique parfois, des politiques. Les leaders politiques, eux, ont l'intention de profiter d'un événement médiatique, intégré à leur programme de campagne. Aussi faut-il s'interroger sur l'importance qu'ils

7. Étant donné les objectifs de notre travail, nous n'avons considéré pour la transcription du corpus que quelques symboles, que nous signalons ci-après. Les participants au programme sont désignés par des majuscules; pour les leaders politiques : JS (José Sócrates), MFL (Manuela Ferreira Leite), JdS (Jerónimo de Sousa), FL (Francisco Louçã); comédien : RP (Ricardo Pereira). Interruption d'un énoncé ou d'un mot par le locuteur: [...]; interprétation d'une interruption par l'analyste : (aaa?); coupes effectuées par l'analyste : [...]; commentaire fait par l'analyste ou présentation d'un événement non verbal : [aaa]; chevauchements : aaa; productions vocales: ahm ; intonation forte: AAA ; les conventions habituelles de ponctuation de l'écrit - point, point d'interrogation, point d'exclamation - sont conservées. La ponctuation et les majuscules sont rétablies dans la traduction française. Le programme est enregistré sur CD-rom. Pour analyser les exemples, nous avons mis les expressions concernées en italique.

8. Selon Kerbrat-Orecchioni (2005, p. 19), «l'ADI a par définition pour objet les divers types de discours qui sont produits en contexte interactif. [...] l'ADI met naturellement l'accent sur les phénomènes dont le caractère interactif est le plus évident». 
accordent à cet événement face au risque d'être victimes de l'humour ou, pire encore, victimes sans humour ou du moins sans savoir-faire. On ne peut qu'être frappé par la place centrale qu'acquiert dans cette campagne la participation des politiques à une émission humoristique. Cela s'inscrit dans un désir d'humanisation de la politique et dans une personnalisation de la campagne où toutes les formations politiques misent sur la personnalité de leur leader. Cette rencontre médiatique, qui a effectivement mobilisé les politiques, leur fournit une occasion de renforcer l'image de proximité familière qu'ils essaient de faire passer partout et, surtout, elle est fondamentale pour créer ou préserver un ethos de personne dite normale, proche des citoyens.

\section{Implications génériques dans la construction de l’interaction. Régulations discursives et relations interpersonnelles}

Les places occupées par les interlocuteurs ne sont que partiellement déterminées par l'espace discursif préexistant à l'interaction; la représentation mentale qu'ils se font de la situation interactionnelle à laquelle ils participent et des rôles sociaux et langagiers qu'ils y jouent leur permet d'en redéfinir les paramètres.

Il faut, d'abord, observer le programme en tant que genre télévisuel. Ce n'est pas, à proprement dire, un talk-show mais plutôt un simulacre humoristique de talk-show9. La campagne électorale et les politiques sont la cible de l'émission, qui se nourrit de faits politiques inusités, ridicules (verbaux ou non verbaux), de stéréotypes, de gaffes, surtout. C'est la construction d'un contenu et d'un point de vue comiques par l'absurde.

Et cette stratégie conditionne aussi l'interaction finale de l'émission, objet de notre analyse. La relation institutionnelle créée est déterminée par les rôles de comédien et de politique des participants, mais il nous faut tenir compte de cette dimension de simulation qui modèle l'interaction, d'une part, et du contenu et des places sélectionnées ${ }^{10}$, d'autre part, pour catégoriser et décrire soit le genre de l'émission télévisuelle, soit l'interaction qu'elle comprend.

Typologiquement, les interactions sélectionnées sont complexes et hétérogènes. D'après la catégorisation fixée à l'avance par les auteurs du programme, nous avons affaire à une interview :

RP : olá [...] é a parte em que temos a entrevista final do programa e o convidado de hoje $[. .$.

9. Sur les caractéristiques de ce phénomène télévisuel, le talk-show, on renvoie à Tolson (2001) et à Charaudeau, Ghiglione (1997). L’hétérogénéité des genres télévisuels est reconnue et «la plupart des genres télévisuels résistent à une description stable» (Martel, 2010).

10. Vion (1995, p. 185) parle à ce sujet de places subjectives : «Il s'agit donc des places « conquises» ou «perdues» et des jeux stratégiques par lesquels les sujets mettent en scène des images d'eux-mêmes.» 
RP : Salut, [...] c'est le moment où nous avons l'interview finale du programme et notre invité d'aujourd'hui [...]

Mais il s'agit surtout d'une catégorisation empirique faite par les participants, qui ne correspond pas forcément aux caractéristiques prototypiques de ce type d'échange langagier. D'ailleurs, s'il est fait référence aux rôles communicatifs d'intervieweur et d'interviewé, c'est justement pour les mettre en question :

RP : muito bem muito bem eu também não aprecio que me chamem jornalista [...]

RP : Très bien, très bien, moi non plus je n'aime pas qu'on m'appelle journaliste [...]

On a plutôt une redéfinition ou transgression du genre, déclenchée par la façon dont les interlocuteurs se positionnent l'un vis-à-vis de l'autre et organisent leur relation interpersonnelle. Certes, la distribution des tours est classique et les séquences du type question-réponse sont les plus fréquentes; l'organisation proxémique est aussi celle d'une interview prototypique, ayant lieu dans un espace organisé autour d'une table, où les participants s'assoient l'un en face de l'autre. Mais il existe des dissonances, qu'il faut signaler. Il ne s'agit pas d'une interaction strictement dyadique : le public est présent dans le studio; il ne parle pas, mais est néanmoins un participant ratifié, il joue le rôle d'auditoire. Les rires et les applaudissements qui ponctuent l'interaction manifestent son adhésion au "spectacle " ${ }^{11}$ et surtout ratifient les rôles que les interlocuteurs se construisent au fur et à mesure du déroulement interactionnel. Il est convoqué explicitement comme participant et destinataire dès le début de chaque interaction :

RP : olá [...] senhoras e senhores a presidente do psd a doutora manuela ferreira leite $[. .$.

RP : Salut [...] mesdames et messieurs, la présidente du PSD, Madame Manuela Ferreira Leite [...]

D’autres données contextuelles, proxémiques, sont aussi à remarquer. Au début de l'interaction, l'invité n'est pas présent, comme il est de règle dans les interviews politiques; il entre en scène après avoir été présenté par l'animateur du programme, et pendant que le public applaudit, comme s’il était une vedette de talk-show. Les invités jouent le rôle de politiques, mais ils ne respectent pas le code vestimentaire toujours formel d'une interview politique à la télévision. Leur décontraction vestimentaire est caractéristique de celle des politiques pendant les meetings, ou lors d'autres contacts avec la population, dans la rue, pendant les campagnes électorales : ils se présentent sans cravate,

11. D’après Chabanne (1999, p. 45), le rire « peut avoir de nombreuses fonctions [...] - II marque évidemment la réception réussie de l'énonciation humoristique». 
la chemise ouverte ou en manches courtes. L'animateur, lui, respecte le code vestimentaire d'un intervieweur politique, mais assume explicitement de n'être pas journaliste et d'en simuler le rôle. Ses interventions mettent en relief son rôle central de comédien, dont le public est le destinataire principal. Il prend la parole et pose des questions pour faire rire. Dans l'exemple suivant, il ne s'agit pas de questionner directement son invité à propos d'une éventuelle baisse des impôts, mais de provoquer le comique, en envisageant la relation institutionnelle entre le gouvernement portugais et les citoyens comme une relation typique du petit commerce, entre un vendeur et un client habituel. C'est donc à un niveau pragmatique que l'humour est construit. Et c'est le remplacement inusité d'un script par un autre qui provoque le nonsense et l'effet comique :

RP : [...] a doutora manuela ferreira leite foi ministra da educação no tempo em que eu era estudante depois era ministra das finanças na altura em que eu entrei no mercado de trabalho e agora pode ser primeira-ministra não acha que eu por ser um cliente antigo devia ter um desconto no irs? [Gargalhadas e palmas do público. Risos MFL.] [...]

RP : [...] Vous avez été ministre de l'Éducation au temps où j'étais étudiant, après vous avez été ministre des Finances au moment où j'entrais sur le marché du travail, et maintenant vous serez peut-être Premier ministre... Vous ne trouvez pas qu'en tant que vieux client, je mériterais une réduction de l'impôt sur le revenu? [Rires et applaudissements du public. Rires de MFL] [...]

La gestion de l'interaction est effectivement partagée parce qu'il s'agit d'un dialogue, mais surtout parce que la gestion des places n'est pas entièrement prévisible et donne lieu à des développements discursifs variables par un ensemble de processus adaptatifs visant la création, en premier lieu, d'un univers de jeu et de connivence. Il est important d'examiner la façon dont l'animateur gère la relation à son invité, coincé entre l'objectif de faire rire en se moquant de lui, et le besoin de préserver un lien de connivence, de ne pas mettre en danger, en somme, la face de chaque invité. La préoccupation de politesse est dominante. L'animateur s'adresse à ses interlocuteurs en utilisant un langage soutenu et poli :

RP : [...] senhor deputado jerónimo de sousa muito boa noite [...]

$\mathrm{RP}:[. .$.$] ontem foi o senhor primeiro-ministro agora a doutora manuela ferreira leite$ vem sugerir [...]

RP : [...] eu queria perguntar-lhe também senhor primeiro-ministro a propósito do desemprego [...]

RP : [...] Monsieur le députéJerónimo de Sousa, bonsoir [...]

RP : [...] Hier, c'était Monsieur le Premier ministre, maintenant c'est vous qui venez me suggérer [...]

RP : [...] Je voudrais vous demander aussi, Monsieur le Premier ministre, à propos du chômage [...] 
Pour mettre son interlocuteurà l'aise, il se construit une image anti-menaçante pour lui en mettant en œuvre des stratégies destinées à éviter la confrontation. Il choisit de dévaluer son propre statut et se présente soit comme un intervieweur sans expérience, soit comme un comédien sans importance. En formulant des actes menaçants pour soi-même, qui inversent apparemment la relation asymétrique et le statut d'expert de l'intervieweur prototypique du genre interview, le comédien occupe une position basse par rapport à son invité. On observe, en convergence avec ce comportement, une complète absence de confrontation. Tout désaccord est évité ou vite réparé :

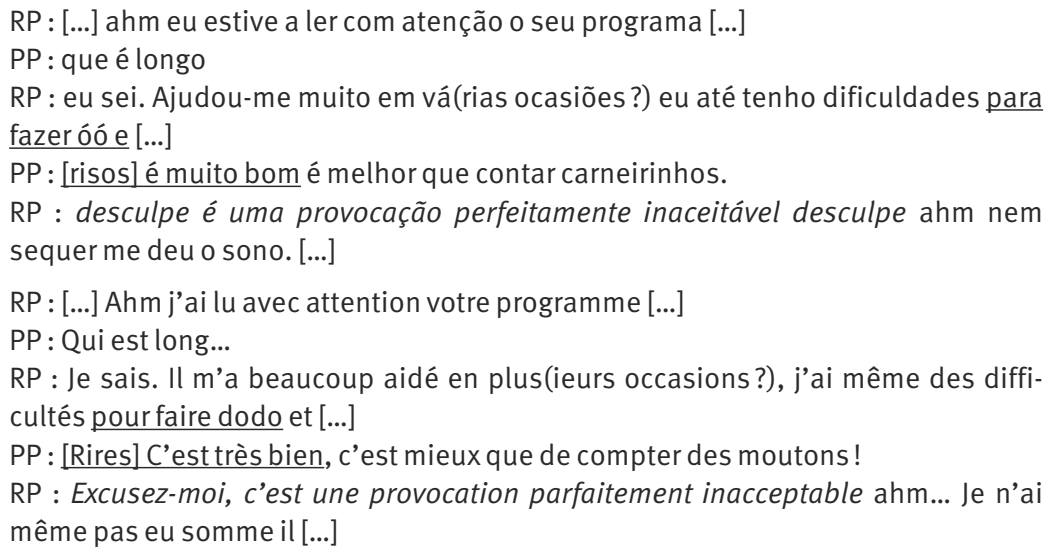

L'animateur manifeste aussi un assentiment systématique et rassurant, ponctué par des signes régulateurs :

MFL : [...] ahm bom para já nas próximas eleições vou votar em mim $\mathrm{RP}:$ muito bem $[\ldots]$

JdS : [...] vou continuar a amar portugal vou ficar em portugal é o meu país de escolha RP : muito bem muito bem $[. . .]^{12}$

MFL : [...] Ahm bon, d'abord aux prochaines élections, je vais voter pour moi-même. RP : Très bien [...]

JdS : [...] Je vais continuer à aimer le Portugal, je vais rester au Portugal, c'est le pays que j'ai choisi [...].

RP: Très bien, Très bien [...].

Toutefois, ce positionnement du comédien est foncièrement ambigu et on ne peut évidemment pas écarter le fait qu'il s'agisse d'une stratégie de politesse piégée, parce que l'espace ainsi accordé à l'invité pour le faire parler fournit aussi l'opportunité de faire rire le public :

12. Dans l'ensemble des interventions réactives, nous avons 47 signes régulateurs d'assentiment: interaction avec JS : 9 ; interaction avec FL : 8 ; interaction avec MFL : 13; interaction avec JdS : 6 ; interaction avec PP: 11. 
RP : [...] senhor deputado jerónimo de sousa em qual destas democracias é que o senhor preferia viver suécia noruega ou coreia do norte? [risos]

RP : [...] Monsieur le député Jerónimo de Sousa, dans quelle démocratie préféreriezvous vivre : en Suède, en Norvège ou en Corée du Nord? [Rires] [...]

Aménager le positionnement discursif, le rapport de places, est une tâche complexe chez les invités, révélatrice des enjeux de la politique. Ils refusent, tous, le statut de cible et prétendent à la réciprocité de places, à la proximité informelle, sans rôles préétablis, statut qui est plutôt caractéristique d'une conversation. Les termes d'adresse utilisés sont révélateurs ${ }^{13}$ :

MFL : [...] eu acho que você deve ter dado um traBAlho tremendo aos seus pais desde que nasceu [...]

JS : [...] você deve ser para aí o sexto apresentador mais elegante do mundo [...]

PP : [...] você lembra-se daquela frase dele está para nascer um primeiro-ministro tão bom como ele próprio não é? [...]

MFL : [...] Je crois que vous avez certainement donné beaucoup de traVAil à vos parents depuis votre naissance [...]

JS : Vous êtes certainement le sixième animateur le plus élégant du monde [...]

PP : [...] Vous vous souvenez de ce qu'il a dit, qu'il n'est pas encore né un Premier ministre aussi compétent que lui, n'est-ce pas? [...]

Ils choisissent par ailleurs des stratégies de politesse positive (KerbratOrecchioni, 1997); l'éloge de l’interlocuteur est un acte de langage récurrent :

JS : mas deixe-me dizer-lhe que você está elegantíssimo [...]

$\mathrm{FL}$ : vocês são imbatíveis no vosso lado [...]

RP : obrigado

FL a mandar bocas são absolutamente imbativeis [...]

JS : Mais permettez-moi de vous le dire : vous êtes très très élégant [...]

$\mathrm{FL}$ : Vous êtes imbattable dans votre domaine [...]

RP : Merci.

FL: Pour ce qui est de charrier les gens, vous êtes absolument imbattable [...]

En outre, et c'est une visée fondamentale, ils se doivent de gagner la confiance du public. L'association entre le caractère informel, d'une part, et la sincérité et le dévoilement de soi, d'autre part, est un lieu commun dont les locuteurs politiques profitent pour établir et légitimer aussi une relation de proximité avec le public. En fait, le politique, qui est la cible dans la première partie du programme ${ }^{14}$, devient alors partenaire grâce à la relation interpersonnelle

13. Il y a plusieurs études sur le terme d'adresse você. Je renvoie à Carreira (2004) : «... la forme pronominale você [...]. Cette forme généralisée au Brésil, sans qu'aucune connotation ne lui soit rattachée, est la forme la plus sensible, au Portugal, aux variations diatopiques et diastratiques... ».

14. Le programme dont MFL est l'invitée commence avec ces mots : «MFL vem a um sítio onde pode sempre dizer que aquilo não era uma gaffe era uma piada » [MFL vient dans un lieu où elle peut toujours dire que ce qu'elle vient de dire n'était pas une gaffe mais une blague]. 
qu'il construit dans le discours à l'égard des autres participants. Il est colocuteur, il cogère l'interaction dans un cadre de connivence dont le public fait partie, et ce positionnement surplombe celui de cible (Charaudeau, 2006). Dans ces interactions humoristiques, on rit avec l'invité, pas de l'invité.

En fait, la construction des contenus renforce ces rapports de places coopératifs bien que complexes. Les questions posées portent sur des sujets déjà parodiés à d'autres moments du programme humoristique, et sur des situations discursives antérieures marquées en quelque sorte par le ridicule. C'est un discours sur des discours ; le dialogisme interdiscursif 15 structure l'interaction et soutient les stratégies à l'œuvre. L'animateur-comédien adopte une stratégie de reprise de ces événements antérieurs, sans confrontation explicite. C'est par des allusions que le comédien rapporte les paroles de ses invités. Donc, bien qu'il s'agisse de la stratégie la plus utilisée, l'allusion met son allocutaire à l'abri d'une agression directe :

RP : [...] se me permite eu voltava às provocações maldosas [...] eu queria perguntarthe se não está para nascer um primeiro-ministro que tenha mais professores contra si do que o senhor? [gargalhadas do público. Risos de JS] [...]

RP : [...] a última pergunta não a incomodo mais se a doutora manuela ferreira leite ganhar as eleições e com o professor cavaco silva em belém estão finalmente reunidas condições para que possamos suspender a democracia durante uns tempos? [gargalhadas do público]

MFL : bem se vê... pela sua pergunta aí se vê que a ironia tem os seus perigos [gargalhadas. RP risos] [...] ${ }^{16}$

RP : [...] Si vous me permettez, je reviendrai aux provocations malveillantes [...] J'aimerais vous demander s'il est en train de naître un Premier ministre qui ait plus de professeurs contre lui que vous? [Rires du public. Rires de JS] [...]

RP : [...] Ma dernière question je ne vous dérange plus, mais si vous gagnez les élections et étant donné que le professeur Cavaco Silva est à Belém, les conditions pour qu'on puisse suspendre la démocratie pendant une certaine période sont finalement réunies? [Rires du public]

MFL : C'est évident... Votre question montre que l'ironie a ses dangers [Rires du public. Rires de RP] [...]

Le comédien pose des questions qui frôlent l'absurde, mais prolongent l'«absurde» déjà présent dans les interventions politiques rapportées. Cette

15. Sur le dialogisme interdiscursif et l'auto-dialogisme, voir Bres, 2005.

16. Le Premier ministre a déclaré en 2009 que, relativement au déficit, il n'était pas encore né de premier ministre qui ait fait mieux que lui. Au moment de l'interview, les professeurs étaient en grève. Cavaco Silva, président de la République - Belém est l’Élysée portugais - et Manuela Ferreira Leite sont tous deux du PSD alors que le président et le Premier ministre ont assez souvent été, depuis 1974, d'orientations politiques différentes. Manuela Ferreira Leite a effectivement dit qu'il faudrait suspendre la démocratie pendant une période de six mois pour que les réformes proposées par le gouvernement soient réalisées. Elle assume ces paroles, qu'elle justifie comme étant de l'ironie. Peut-être le comédien et le public n'y croient-ils pas vraiment, ou considèrent-ils qu'elle s'en est bien tirée. 
mise en parallèle est en soi menaçante. Toutefois, le côté dialogique de l'humour, que nous avons déjà souligné, permet aussi aux politiques de suspendre leur responsabilité en mettant en scène un énonciateur qui s'éloigne du point de vue rapporté. En prenant leurs distances vis-à-vis du sujet ridiculisé, ils s'assimilent au point de vue critique du comédien (et du public), et ils se construisent en même temps une image attirante de savoir-faire. Chaque locuteur-invité met en scène un énonciateur qui est en accord avec l'énonciateur critique et qui s'éloigne des situations maladroites que le comédien-animateur convoque. Dans l'exemple ci-dessous se manifeste même un jeu de points de vue repris et mis en scène par auto-dialogisme, en même temps qu'un éloignement moqueur vis-à-vis de ce point de vue, notamment par l'usage d'un verbe au conditionnel, qui permet au locuteur de sauver la face; cela le rapproche du point de vue de son interlocuteur et le met à l'abri de la raillerie ${ }^{17}$ :

RP : ahm queria fazer-lhe uma pergunta sobre enfim sobre a sua vida lá fora de todos de todos os líderes europeus e outros que tem conhecido qual é aquele que é digamos como é que eu hei... qual é o mais porreiro pá [gargalhadas] qual é o mais [...]

IS : [...] se eu tivesse portanto de definir aqueles que acho mais porreiro pá era destes que eu falaria. [...] $]^{18}$

RP : Ahm je voudrais vous poser une question sur... enfin, sur votre vie à l'étranger : de tous les leaders européens ou autres que vous avez connus, quel est celui... disons... je sais pas comment di... Quel est le plus cool, mec [rires du public], quel est le plus [...]

IS: Si je devais donc définir ceux que je trouve les plus cool, mec, ce serait de ceuxlà que je parlerais [...]

Les politiques se construisent ainsi un ethos positif. L'adhésion au registre ludique du comédien leur permet de faire preuve d'humour et de s'assurer, par conséquent, ce statut de réciprocité, d'égalité dans l'interaction et de proximité avec l'électorat qu'ils cherchent depuis le début. Et le public confirme la réussite de la démarche par des rires et des applaudissements.

En fait, les deux interlocuteurs s'engagent - certes, par des voies différentes - dans un contrat de complicité dès l'ouverture de l'interaction. Il existe, du moins apparemment, un alignement des prises de paroles, un entrelacement des interventions de l'un et de l'autre par des reprises consensuelles. La reprise en écho de l'intervention de l'interlocuteur souligne cette continuité et cette conformité de points de vue. Dans le premier exemple ci-dessous, l'interviewé interrompt le comédien pour faire de l'humour, en reprenant une situation humoristique d'une émission antérieure; l'animateur l'accepte et reprend

17. La stratégie de l’allusion est partout : «porreiro, pá!», c’est ce que José Sócrates a susurré à Durão Barroso à la fin du sommet de Lisbonne, en 2009. Les micros étaient encore ouverts...

18. Pá est un terme d'adresse très familier, ou alors une sorte de tic ponctuant un discours oral. 
à son tour la même expression. Dans le deuxième exemple, l'interviewé renforce le point de vue du comédien. Il semble qu'on soit face à une conversation coopérative, loin des menaces d'une reprise potentiellement railleuse ${ }^{19}$ :

RP: eu eu [...] um dos pontos fundamentais do seu programa é o apoio às pequenas e médias empresas eu [...] acho com franqueza que há aí uma nota de eleitoralismo PP : nano micro

$\mathrm{RP}$ : nano micro ah curiosa [...] curiosamente [...]

PP : [...] Je... Je [...] Un des points fondamentaux de votre programme, c'est l'appui aux petites et moyennes entreprises... Je... crois franchement [...]

PP : Nano micro

RP : Nano micro... ah curieuse... curieusement [...]

$\mathrm{FL}:[. .$.$] as grandes empresas as pequenas empresas [...] as micro empresas as nano$ empresas todas elas são precisas desde que dêem emprego

RP : eu vejo com agrado que a nossa peça sobre nano mini micro pequenas e médias empresas faz cultura política

FL: sim faz cult [...]

$\mathrm{RP}:$ faz cultura política $[. . .]^{20}$

FL : [...] Les grandes entreprises, les petites entreprises [...], les entreprises micro, les entreprises nano... sont toutes nécessaires pourvu qu'elles créent des emplois. $\mathrm{RP}$ : Je vois avec plaisir que notre pièce humoristique sur les nano-mini-micropetites et moyennes entreprises fait la culture politique.

$\mathrm{FL}:$ Oui, cela fait la cult [...]

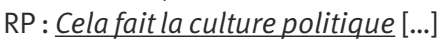

Mais il faut tenir compte du fait que le politique-invité est toujours la cible de l'humour du comédien malgré tout ce travail de figuration que comédien et politique entreprennent pour sauver la face (celle de l'invité, en particulier).

Parallèlement, le rôle institutionnel des politiques est toujours présent en dépit du ton ludique dominant. D’abord, c'est par rapport au public que les politiques passent du ludique au sérieux. Tous les invités profitent du moment pour faire passer des messages politiques explicites. Ce sont des modules (Vion, 1992, p.149) de discours politique qu'ils prennent le soin de signaler:

PP : [...] bem só um parênteses acho eu porque eu acho que com fome não se brinca [...] isso é objetivo é verificável agora fechando este parênteses [...]

PP : [...] Bon, juste une parenthèse : je crois parce qu'on ne rigole pas avec la faim [...] cela est objectif, c'est vérifiable. Maintenant je ferme cette parenthèse [...]

19. Ce versant coopératif et poli est structurant de chaque interaction. Il n'y a pas de dérision telle que la définit Bonnafous (2001, p.53) : «la “dérision”, c'est-à-dire [...] cette association de l’humour et de l'agression ".

20. Pendant la campagne électorale, le besoin d'appuyer politiquement et économiquement les petites et moyennes entreprises portugaises (contre les grandes entreprises internationales) est devenu une sorte de «leit-motiv» pour tous les leaders politiques. Les Gato Fedorento ont repris ce thème en mettant en scène un politique qui se considérait comme différent des autres parce qu’il défendait les «nano, mini, micro entreprises». 
On observe quand même une simplification du domaine du politique, souvent réduit à des récits personnels, étonnants peut-être, racontés dans un registre courant, oralisant, marqué, par exemple, par l'usage du déterminant défini devant le nom propre et de la deuxième personne du singulier comme terme d'adresse dans une conversation avec le Premier ministre italien, rapportée par l'interviewé :

JS : [...] lembro-me bem quando conheci o berlusconi eu conheci-o [...] o primeiro conselho que ele me deu foi o seguinte eu estava com um fato com três botões [...] $e$ ele disse não deves apertar os dois o de cima e o do meio para ficares mais elegante e depois disse-me no final o seguinte sabes podes acreditar em mim porque eu já fui alfaiate coisa que eu não sabia [gargalhada do público] [...]

JS : [...] Je me souviens bien du moment où j'ai fait connaissance de Berlusconi [...] Voilà le premier conseil qu'il m'a donné: je portais une veste de costume à trois boutons [...] et il m'a dit : tu ne dois pas boutonner les deux premiers pour être plus élégant, et ensuite il m'a dit ceci : tu sais, tu peux me faire confiance car j'ai déjà été tailleur, ce que je ne savais pas [rires du public] [...]

Cette «petite politique» s'éloigne des modules identifiés ci-dessus. En quête d'une image populaire, humanisée, les politiques acceptent volontiers le mélange du public et du privé, du sérieux et du bouffon.

O Gato Fedorento esmiúça os sufrágios est un phénomène marquant et complexe, signe d'un versant frivole de certaines pratiques discursives de la politique portugaise.

Les interactions verbales, qui sont au centre de notre analyse, posent la question cruciale du rapport de la politique aux médias télévisuels et aux programmes de divertissement en particulier. S'agissant d'interactions relevant à la fois des sphères d'activité médiatique et politique, il existe des tensions sociales et discursives qu'il faut gérer soigneusement, malgré le caractère marqué d'interaction non conflictuelle assuré par le travail systématique de figuration et toutes sortes de comportements coopératifs coconstruits par les interlocuteurs. En l'absence d'un cadre générique déjà connu et stable, ceux-ci construisent et négocient le genre discursif au fur et à mesure de leurs interventions. Ce travail de construction discursive est à relier au dynamisme et aux spécificités de la relation interpersonnelle qu'ils élaborent. Le caractère dialogique des interactions est fondamental pour leur succès.

En ce qui concerne les politiques, ces démarches servent les objectifs de visibilité privilégiés par les discours de campagne. Mais en même temps, le cadre humoristique du programme met à l'épreuve l'image personnelle des politiques, nettement amplifiée en conjoncture électorale, et questionne surtout ces formes nouvelles de faire de la politique. Dans ce cas, le politique se fait prendre par l'humour. 
En termes strictement politiques, la participation à ce genre de programmes télévisuels est une action de campagne, qui se prétend légitime et même sérieuse, afin de consolider une relation de proximité avec le peuple et d'obtenir ses suffrages. Mais revenir au peuple semble impliquer la spectacularisation du politique. Indépendamment de leur positionnement à droite ou à gauche dans le panorama politique portugais, tous les politiques invités se sont construit des places subjectives de non-spécialistes, marquées par un registre familier, sans pour autant refuser leur rôle de politiques. Dans ce mélange de fonctions, ce sont surtout les petits épisodes de la vie politique qui sont les plus visibles. L'attitude populiste que l'ambiance de campagne électorale encourage devient vite peopolisme (Rabatel, 2011) et la politique se donne en spectacle.

La relation interpersonnelle construite en interaction est aussi piégée à un autre niveau. En ce qui concerne l'incursion dans cette émission d'humour, les invités construisent in praesentia une relation de proximité et de symétrie avec le comédien-animateur, faussée en fait par l'intégration in absentia dans l'événement discursif global du programme humoristique, où les invités ne sont que les traditionnelles cibles d'un humour mordant.

\section{Références}

Bonnafous Simone, 2001, «L'arme de la dérision chez J.-M. Le Pen », Hermès, n²29, p. $53-63$.

BRES Jacques, 2005, "Savoir de quoi on parle : dialogue, dialogal, dialogique; dialogisme, polyphonie ", Dialogisme et polyphonie, approches linguistiques. Actes du colloque de Cerisy, J. Brès, P. Haillet, S. Mellet, H. Nolke, L. Rosier éd., Bruxelles, De Boeck-Duculot, p. 47-62.

ChabANne Jean-Charles, 1999, "Verbal, paraverbal et non-verbal dans l'interaction humoristique », Approches du discours comique, J.-M. Defays, L. Rosier éd., Paris, Mardaga, p. 35-53.

CARreira Maria Helena, 2004, «Les formes allocutives du portugais européen. Évolutions, valeurs et fonctionnements discursifs», Paris, Instituto Cervantes, http:// cvc.cervantes.es/lengua/coloquio_paris (consulté le 12 mars 2010).

Charaudeau Patrick, 2006, «Des catégories pour l'humour?», Questions de communication, n' 10 , p. 19-41.

- 2010, "O discurso propagandista : uma tipologia», Análises do discurso hoje, I. Machado, R. Mello éd., vol. III, Rio de Janeiro, Nova Fronteira, p. 57-78.

- 2011, "Réflexions pour l'analyse du discours populiste», Mots. Les langages du politique, n97, p.101-116.

Charaudeau Patrick, Ghiglione Rodolphe, 1997, La parole confisquée. Un genre télévisuel : le talk-show, Paris, Dunod.

Kerbrat-OrecchionI Catherine, 1997, "Variations culturelles et universaux dans le 
fonctionnement de la politesse linguistique», Le Dialogique. Colloque international sur les formes philosophiques, linguistiques, littéraires et cognitives du dialogue, D. Luzzatti, J.-C. Beacco, R. Mir-Samii, M. Murat, M. Vivet éd., Berne, Peter Lang, p.151-159.

- 2005, Le Discours en interaction, Paris, Armand Colin.

MARQUeS Maria Aldina, 2005, "Os apartes no discurso político parlamentar : vozes off », Ciências da Linguagem. 30 anos de investigação e ensino, M.A. Marques, E. Koller, J. Teixeira, A. Sampaio éd., Braga, CEHUM, p. 193-216.

MARTEL Guylaine, 2010, "Construction de l'image médiatique des politiciens. Des stratégies en plusieurs genres pour toutes les identités», Les médias et le politique. Actes du colloque "Le français parlé dans les médias», J. Burger, J. Jérôme, R. Micheli éd., Lausanne, Centre de linguistique et des sciences du langage, http:// www.unil.ch/clsl/page81503.html (consulté le 12 mars 2012).

MCNAIR Brian, 2011, An Introduction to Political Communication, Londres, Routledge.

RABATEL Alain, 2011, "Deux modes de représentation idéologique people du pouvoir, lors du premier anniversaire de la présidence de N. Sarkozy », Semen, nº 30, http:// semen.revues.org/9010 (consulté le 21 mars 2011).

Tolson Andrew éd., 2001, Television Talk Shows. Discourse, Performance, Spectacle, Londres, L. Erlbaum.

TSAKONA Villy, 2009, «Humour and image politics in parliamentary discourse. A Greek case study », Text and Talk, n² 29-2, p. 219-237.

VION Robert, 1992, La communication verbale. Analyse des interactions, Paris, Hachette.

- 1995, "La gestion pluridimensionnelle du dialogue», Cahiers de linguistique française, $\mathrm{n}^{\circ} 17, \mathrm{p} .179-202$.

- 1996, «L'analyse des interactions verbales », Les Carnets du Cediscor, n 4 , http:// cediscor-revues.org/34 (consulté le 6 mars 2012). 